\title{
Research on Load Balance based on Fujian Meteorological Cloud Platform
}

\author{
Zhu Tianfu1, a, Yang Zhiyong1,a and Zhang Lei2,a \\ ${ }^{1}$ Fujian Provincial Meteorological Information Center, China \\ ${ }^{2}$ Office of Fujian Provincial Meteorological Bureau, China \\ a191546861@qq.com
}

Keywords: Load balancing; Cloud computing; Virtual machine load; Resource utilization.

\begin{abstract}
Load balancing problem is one of the hot issues in cloud computing. Based on Fujian meteorological cloud platform, the establishment of the model carries out quantification the load status and cluster resource utilization rate of virtual machine, and the improved LC algorithm is proposed with the meteorological data features. The verification shows that this algorithm can improve the load balance of resource utilization and cloud computing.
\end{abstract}

\section{Introduction}

In order to better meet meteorological, water conservancy, aviation, forestry and other needs, this paper can realize the meteorological disaster monitoring of the rural areas, coastal areas, major rivers, major strategic economic zones and geological disasters prone areas, to improve the comprehensive monitoring and early warning, resilience and disaster reduction of the weather disaster [1,2]. At the same times, it can avoid aviation, water conservancy and other departments on weather radar observation network redundant construction, to maximize the investment benefits. In the province, we can share weather radar observation network information, Fujian Provincial meteorological bureau builds a new generation of data cloud computing center platform.

Load balance is the basic element of cloud computing, and it is the key link of server cluster. Compared to traditional single process load balancing framework, a multi process architecture can take full advantage of cloud computing parallel processing ability to improve overall system performance in cloud computing [3-5]. Load balancing technology is a kind of strategy, it can make multiple servers or multiple links shared some heavy computation and I / Q task, so as to lower the cost of eliminating the network bottleneck and improve the reliability and flexibility of the network. Load balancing technology not only can maintain the load balance distribution in the network system, but also can maintain the efficient operation of the network system, so it is the important technology to guarantee the high performance of the network system. In this paper, we mainly study the parallel processing efficiency of the "cloud computing" with the appropriate load balance algorithm in the special background of meteorological data.

\section{Load Balancing Mechanism and its Algorithm under the Cloud Computing}

Load balancing problem description under the cloud computing. Cloud computing and core concept are resource pool, and scheduling algorithm is the core of cloud computing resource allocation, cloud computing scheduling is load balance between the virtual machine in data center.

Cloud computing task allocation is divided into three levels: task request layer, resource management, task execution layer. The task request layer is facing the user, realizing the user and system interaction obtain the user's request; resource management layer cuts into several sub tasks with logic independent by using the MapReduce tasks, and then sub tasks assign to appropriate the physical machine virtual node according to the load balancing mechanism to parallel processing [6]. This paper only discusses the situation of independent parallel processing after cutting sub tasks; resource management layer is the key to achieve reasonable scheduling of cloud computing resources. Under the cloud computing environment, the physical machine is cut into a plurality of virtual 
resources by using virtualization technology, and the virtual resource is on-demand distribution and automatic growth, but the distribution amount and automatic gain reduction cannot exceed the physical machine itself limit [7]. In a cloud, the number of virtual nodes is segmented, parallel processing resource management layer divides the same number of sub tasks, and it is asked to perform the task execution time as small as possible.

Load balancing mechanism has a fundamentally different in the cloud platform and the cluster environment. In a cluster environment, the task processing is based on the actual physical host, if the acquired host is too much, it is not only invested large; when the load is too low, it will cause resource waste serious; if host is too little, load exceeds the total load capacity, it will cause great impact on QoS system, so the traditional load balancing is a rigid load balancing. The cloud platform virtual resources are a dynamic resource environment, which can add or reduce the virtual machine according to the actual load change.

Selection load balancing algorithm. There are many load balancing algorithms that are round robin algorithm (RR) algorithm, weighted round robin (WRR) algorithm, least connection (LC) algorithm and weighted least connection (WLC) algorithm [8].

(1) RR algorithm: the customer request can distribute to each member server, RR mode can be simply interpreted as a random choice. This algorithm takes the switch server as the same and does not consider each server connection number and response time, and it is equivalent to randomly select the server of business in the serve. However, this algorithm is randomly chosen for server, so it is not ideal for server load balancing in some cases.

(2) WRR algorithm: based on the RR algorithm, according to establish of the operation speed of each server and the number of connection different abilities in the server group, the weights of each server in the server group are added to the response, and then according to the weight of the processing to improve the algorithm load balancing ability. WRR algorithm is suitable for each server with different processing capability. In the algorithm, each server is assigned a weight, the weight is an integer, it is shown that the corresponding to the processing power of the server. In the server set up or release the connection, the server weight values will change. The WRR algorithm chooses the best response server to provide service according to the different weight of each server, which can simulate better server load balancing process.

(3) LC algorithm: network connection point have at least the number of connected servers, this is a dynamic switching algorithm, which needs to dynamically calculate the number of connections established by each server, and then to dynamically select the corresponding server according to the obtained results. In the virtual server, all server processing ability and the establishment of connection difference are smaller, if the LC algorithm in the number of requests varied greatly, it can very good request distribution, this is due to the arrival of switch requests will not be pointed at a single server caused by overweight load of the server. However, the LC algorithm does not consider the load imbalance caused by the server performance difference with different processing capabilities servers,

(4) WLC algorithm: it is the improvement of LC algorithm. In the WLC algorithm, each server will have a weight value, the server weight value is higher, and indicating that the number of established server connections has lower percentage of the total number of established server connections, server has not many connected and still has considerable service ability. The switch gives the weight for each server, the switch is according to the weight value, the network connection is divided the server. WLC algorithm is to select the server according to established server connection number and weight value ratio, the algorithm finds the smallest ratio server to establish a connection, this ratio shows the current server load condition.

\section{Construction of the Load Balance Model under the Fujian Meteorological Cloud Platform}

Fujian meteorological cloud platform. Fujian meteorological cloud platform is an IaaS cloud platform of heterogeneous resource type, it not only can support access types Web services, but also support data query service based on information service as the core [9]. For the consideration of the application, the platform resource selection decides that the cloud platform needs the server of the $\mathrm{X}$ 86 schemas and the server of the RISC architecture, so it is an IaaS cloud platform of heterogeneous 
resource type, the platform needs to provide high available resources for different service levels, and the business types of its support are also diverse. Cloud platform users carry out service for each business unit and the council. The reliability, availability, and security requirements of the infrastructure resources provided by the cloud platform are far higher than the general service levels provided by public cloud services.

The platform uses automation technology to improve the platform's flexibility and efficiency, its support business will gradually increase, and the size of the IaaS cloud platform will grow with the expansion of the business scale and the increase of, business types. In order to effectively utilize resources to carry out rapid deployment and adjustment, we should improve the management level of the platform and reduce the management cost, and we also should use automation technology and standardization process, the platform can quickly provide resources according to the business requirements and rapid adjustment resource according to the business development, and business flexible can also share IAAs cloud platform resources.

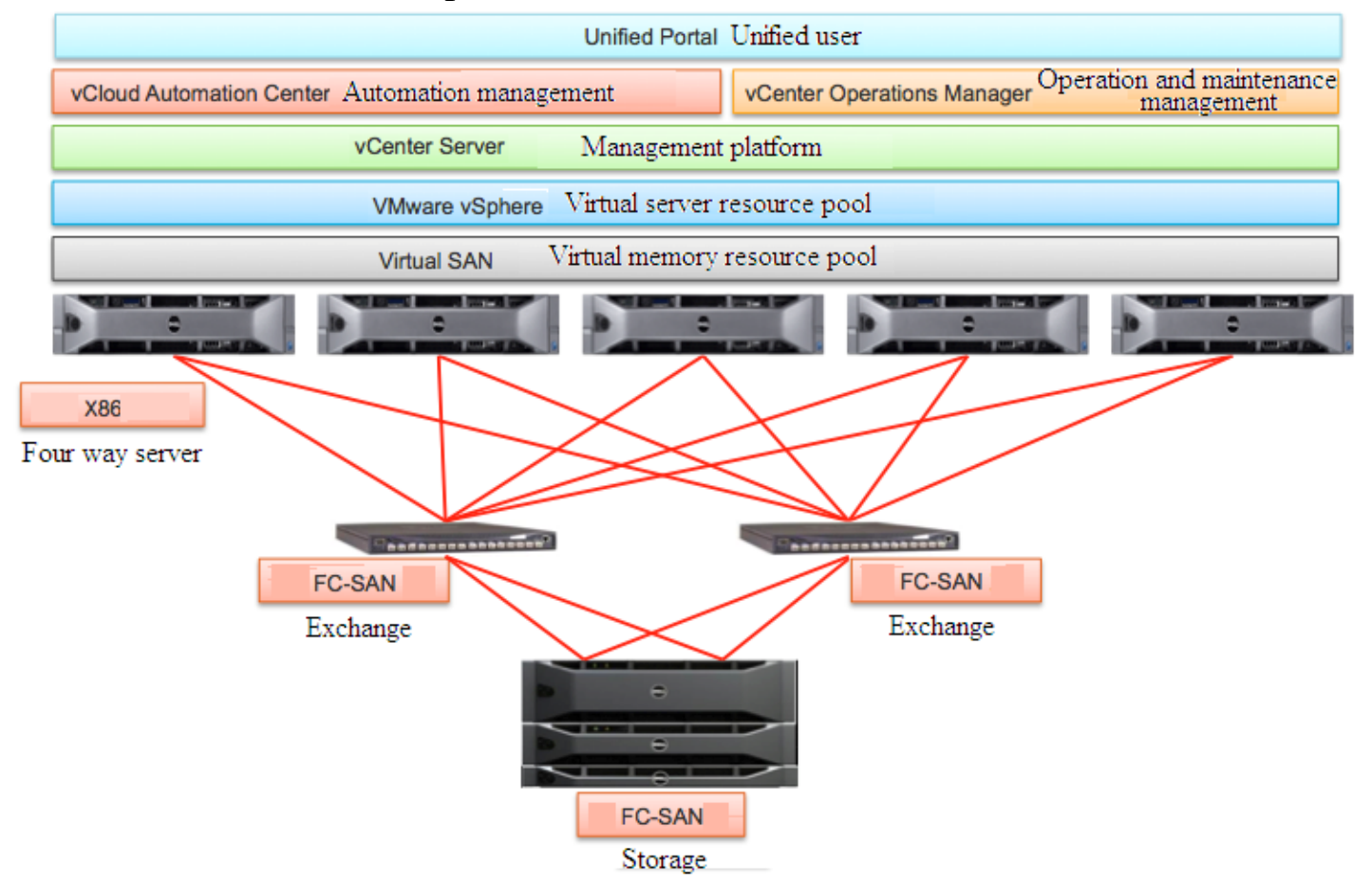

Fig. 1 Fujian meteorological cloud platform system architecture

Load balancing algorithm based on application weight. For load balancing, we should quantify the processing for each server. For the convenience of study, hardware only considers the positive correlation ratio among CPU, hard disk and memory value; the load balance types of computer is divided into computational load and memory load. Due to the hardware configuration of the computer, it determines the load capacity of the computer in a large extent, and software configuration determines the computer service type, so assumption hardware configuration and load are the linear correlation.

Firstly, the CPU utilization of each virtual machine is read from the cluster, disk read rate is respectively for $P c$ and $P d$, and then computing the $C$ value and $s$ value. Set that $C$ is the computational load; $\mathrm{s}$ is storage type load as shown in the following formula:

$$
\begin{gathered}
C=f(x)=\left(\begin{array}{c}
0, x<50 \\
1,50 \leq x<65 \\
2,65<x
\end{array}\right. \\
S=g(x)=\left(\begin{array}{c}
0, x<60 \\
1,60 \leq x<80 \\
2,80<x
\end{array}\right.
\end{gathered}
$$


Due to the different tasks, there are different resource requirements, this paper proposes a load balancing algorithm based on application weights. In the meteorological service, there are many relatively fixed pattern algorithms, and this model assumes that a given cloud computing system introduces to calculate the weight list $W$, it expresses the virtual machine load index weights in the calculation of the model used by evaluation task of the resource requirements. When the virtual machine are presented to calculate the resource, we will be substituted into the model weights, load equalization algorithm contrasts each virtual machine current calculation resource state according to the weights of the calculation model to carry on the selection. As shown in Figure 2, it is LC algorithm with weight to ensure the processing ability of the server, and it can obtain more connection tasks, to some extent, the server can avoid the low processing power due to the task accumulation and paralysis of the situation.

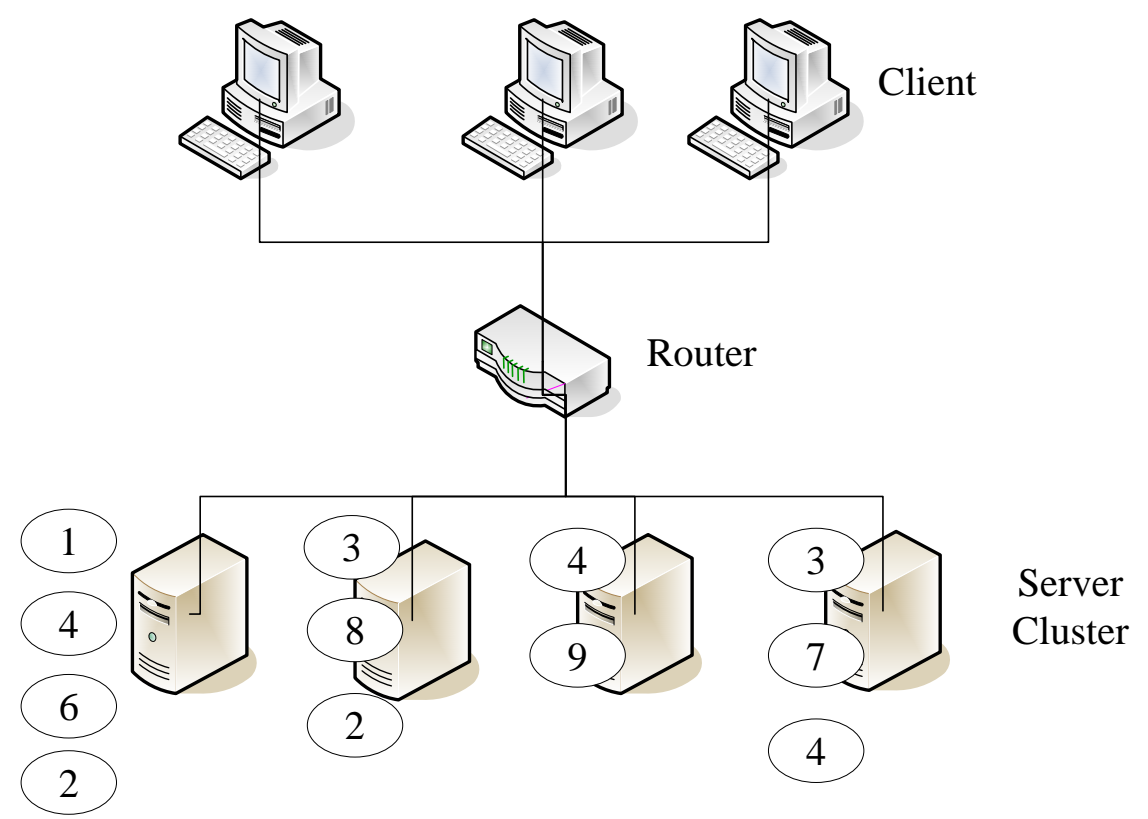

Fig. 2 Weighted LC algorithm

$W i$ indicates the weight of the load application $i$ :

$$
W=\{W i \mid 1 \leq i \leq p\}
$$

$F k$ represents the load of the virtual machine $k$, it evaluates the current load status of the virtual machine.

The resource utilization of virtual machine cluster in load index $i$ is defined by $\mathrm{Si}$ :

$$
S i=\frac{\sum_{i=0}^{i=n} W i}{F k}
$$

\section{Experimental Results Analysis}

In order to verify the effectiveness of the method, this paper simulates the simulation environment under the meteorological cloud platform in Fujian to carry on the experiment study, its main goal is to verify the validity of the method in the cloud computing. At initialization, the virtual machine is requested for resource requests, the amount of the system's request is increased linearly, and then and the load value of the response is calculated. Before and after improved algorithm were tested, the experimental results are shown in Figure 3, the response time of improved algorithm has been obviously increased with the amount of request increased; the average response time after improved algorithm is relatively stable, indicating that the improved algorithm can effectively improve the system resource utilization rate of load balancing. 


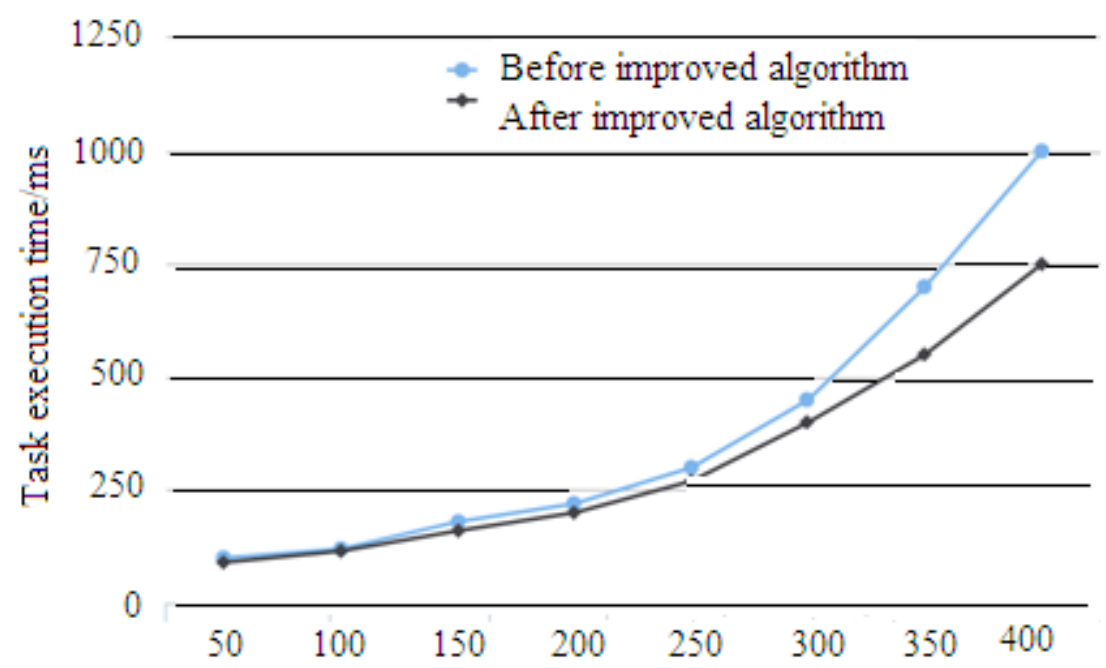

Fig. 3 Experimental tests results before and after improved algorithm

\section{Summary}

This paper introduces a kind of load balancing strategy mechanism based on Fujian Provincial meteorological cloud platform, to study the resource utilization under the improved algorithm and improve the utilization of hardware resources in the simulated environment. With the increase of the number of users cloud platform, people are higher and higher requirements for its stability, compatibility and fault tolerance, how to meet will be the next step to study the content.

\section{References}

[1] Chinang M L,Yang Chenyu,lien S L.Kernel Support for Fine-grained Load Balancing in a Web Cluster Providing Streaming Service[C]//Proc.of the 12th International Conference on Algorithms and Architectures for Parallel Processing. Fukuoka, Japan: Springer, 2012: 458-472.

[2] P.J. Y.. Load Balancing Mechanism for Qos-aware Cloud Computing Using Eucalyptus Platform [EB/OL], 2013, 7(10):79-85.

[3] J.H. H., J.H. G., G.F. S.,et al. A Scheduling Strategy on Load Balancing of Virtual Machine Resources in Cloud Computing Environment[C]// Proc.of the 3rd International Symposiumon Parallel Architectures,Algorithms and Programming.Dalian,Chain:[s.n], 2013:89-96

[4]B. Z., J. G., J.Q. A.. Cloud loading balance algorithm[C]//Information Science and Engineering.Hangzhou,Chain:[s.n], 2013(2):5001-5004

[5]G.J. H., L.J. D.. Data mining principle and its algorithm. Tsinghua university press, 2014:109-118.

[6]Y.P. Z.. Association rules mining and the development. University of electronic science and technology, 2013, 37(7): 113-116.

[7]W.W. C., W.J. C.. The data warehouse and data mining. Beijing: people's posts and telecommunications publishing house K. Elissa, “Title of paper if known,” unpublished.

[8] M. F., X.F. M.. Data mining: concepts and techniques. Beijing: mechanical industry press, 2014: 129-133.

[9]J.W., M. K.. Data mining concepts and techniques. Second Edition. Elsevier Inc, 2013: 111-127. 